[Agr. Biol. Chem., Vol. 31, No. 7, p. 802 808, 1967]

\title{
Sedimentation Properties of Dextran Sulfate-Low Density Lipoprotein Complexes
}

\author{
By Masanobu Janado* and Toshiro Nishida \\ The Burnsides Research Laboratory, University of Illinois, Urbana, Illinois, U.S.A. \\ Received February 27, 1967
}

\begin{abstract}
The nature of interaction between dextran sulfate and the human plasma low density lipoproteins of $S_{f} 0-10$ was investigated in high density media of glycine and glucose. The soluble complex formation between the two components was manifested by sedimentation of the lipoproteins along with dextran sulfate in the glycine and glucose media of density 1.063. The addition of sodium chloride to the mixture caused dissociation of the complex: during subsequent ultracentrifugation, flotation of lipoprotein and sedimentation of dextran sulfate occurred. However, when the complex is in the acidic glycine medium ( $\mathrm{pH} 4.0$ ), the addition of sodium chloride did not induce dissociation of the complex.

Both the solubility and the size of the complex were greatly influenced by the ratio of the two components in solution. At low relative concentrations of dextran sulfate, insoluble aggregates were formed; but the aggregates disintegrated into soluble units upon increasing the dextran sulfate concentrations. From the sedimentation patterns of dextran sulfate lipoprotein mixtures at various ratios, it was possible to estimate the ratio of the two components in the complex. In the presence of excess dextran sulfate a composite biphasic Schlieren diagram was produced as a result of the unusual Johnston-Ogston effect.
\end{abstract}

Recently, considerable attention has been directed toward the release of an enzyme called "lipoprotein lipase" into circulation upon intravenous injection of heparin or other sulfated polysaccharides. ${ }^{11}$ Although the nature of enzymic reaction and subsequent clearance of lipemic sera has been widely recognized, the exact mechanism by which sulfated polysaccharides cause the release of this enzyme has yet to be elucidated. Since sulfated polysaccharides are known to interact with various plasma components, ${ }^{2 !}$ a thorough investigation of their physico-chemical inter-

* Present address: Faculty of Education, Kagoshima University, Kagoshima, Japan.

1) D.S. Robinson, "Advances in Lipid Research," ed. by R. Paoletti and D. Kritchevsky, Academic Press, New York, Vol. I, p. 133 (1963).

2) D. G. Cornwell and F. A. Kruger, J. Lipid Res., 2, 110 (196I). actions may be of fundamental importance in understanding the role of sulfated polysaccharides in the release of lipoprotein lipase and subsequent clearing reactions.

The interaction of lipoprotein and sulfated polysaccharide leads to the formation of either soluble or insoluble complex..$^{3 \sim 7}$ The type of complex formed is known to be determined primarily by nature of sulfated polysaccharide

3) J. L. Oncley, K. W. Walton and D. G. Cornwell, J. Am. Chem. Soc., 79, 4666 (1957).

4) W. W. Briner, J. W. Riddle and D. G. Cornwell, J. Expt. Med., 110, 113 (1959).

5) P. Bernfeld, M. E. Berkowitz and V. M. Donahue, J. Clin. Invest., 36, 1363 (1957).

6) H. N. Anatoniades, J. L. Tullis, L. H. Sargeant, R. B. Pennel and J.L. Oncley, J. Lab. Clin. Med., 51, 630 (1958).

7) P. Bernfeld, "The Lipoproteins, Methods and Clinical Significance,"' ed. P. Homburger and P. Bernfeld, S. Karger, Basel, p. 24 (1958). 
and lipoprotein, the metal ions present, and the $\mathrm{pH}$ and ionic strength of the medium. ${ }^{2,8 \sim 111}$

We have recently studied the interaction between dextran sulfate and low density lipoprotein in phosphate buffer of $\mathrm{pH} 7.4$ and ionic strength $\mu 0.1$ by means of analytical ultracentrifugation. ${ }^{121}$ The results indicated that the ratio of the two components, dextran sulfate and low density lipoprotein, is a decisive factor in the distribution of various molecular species of the complex.

The present experiment was designed to investigate the physico-chemical properties of the soluble dextran sulfate-lipoprotein complexes in high density glycine or glucose media with particelar emphasis on the sedimentation behavior of the complexes.

\section{MATERIALS AND METHODS}

The sodium salt of dextran sulfate (DS), with sulfur content of $17.1 \%$ which corresponds to 1.9 sulfate groups per hexose unit, was prepared from dextran (average molecular weight of 180,000 ) according to the method of Ricketts. ${ }^{13)}$

The human plasma low density lipoprotein (LP $S_{f}$ 0-10) was prepared from citrated whole blood by using a Spinco Model L centrifuge. After removal of chylomicron and very low density lipoprotein of the $S_{f}$ 20-100 class by initial centrifugation, ${ }^{14}$ the solution density of the plasma was adjusted to 1.063 by addition of glycine in the following manner: to $50 \mathrm{ml}$ of plasma freed of chylomicron and very low density lopoprotein was added $15.25 \mathrm{~g}$ of glycine; the mixture was diluted with approximately $30 \mathrm{ml}$ of water to dissolve the glycine, and the final volume of the solution was brought to exactly $100 \mathrm{ml}$ with water. The LP fraction was isolated by ultracentrifugal flotation at 30,000 r.p.m. according to the

8) P. Bernfeld, V. M. Donahue and M. E. Berkowitz, J. Biol. Chem., 226, 51 (1957).

9) P. Bernfeld, J.S. Nisselbaum, B.J. Berkeley and R. W. Hanson, ibid., 235, 2852 (1960).

10) E. Boyle and R. V. Moore, J. Lab. Clin. Med., 52, 272 (1959).

i1) H. Sugano, J. Biochem. (Tokyo), 46, 549 (1959).

12) M. Janado and T. Nishida, J. Lipid Res., 6, 331 (1965).

13) C. R. Ricketts, Biochem. J., 51, 129 (1952).

14) G. A. Gillies, F. T. Lindgren and J. Cason, J. Am. Chem. Soc., 78, 4103 (1956). method of Gofman et al.15) The LP fraction thus obtained was dialyzed against the glycine solution of density 1.063 at $5^{\circ} \mathrm{C}$ for three days with daily change of the external solution. The lipoprotein fraction in glucose medium of density 1.063 was prepared in a similar manner with the use of $16.00 \mathrm{~g}$ of glucose for the density 1.063. The glucose solution of density 1.063 was also used for dialysis.

The LP concentration was estimated by determining the total lipid and protein contents. ${ }^{12)}$ Cholesterol and phosphorus contents of the LP fraction were determined by the methods described by Sperry et al.16) and Fiske et al., ${ }^{17)}$ respectively.

Because of the formation of pronounced density gradients of glycine and glucose, it was suspected that the composition of the two LP fractions might be different from that prepared in the standard sodium chloride medium.15) However, all preparations had essentially identical compositions within experimental errors. Total lipid content ranged from 78 to $79 \%$; protein from 20.6 to $21.8 \%$; total cholesterol from 34 to $35 \%$; and lipid phosphorus from 1.03 to $1.09 \%$.

Sedimentation coefficients $\left(S_{20, w}^{\circ}\right)$ of DS and LP were determined in phosphate buffer ( $\mathrm{pH} 7.4, \mu=0.1$ ); and flotation rate $\left(S_{f}\right)$ of the LP in sodium chloride solution of density 1.063. Intrinsic viscosities ([ $\eta]$ ) were measured in a capillary tube viscometer with a water flow time of 250 seconds. Density measurements for estimating the apparent specific volumes (v) were made in pycnometers of about $5 \mathrm{ml}$ capacity. All determinations were made at $20^{\circ} \mathrm{C}$.

The complex formation between LP and DS was carried out in glycine or glucose media of density 1.063. The formation of soluble or insoluble complexes was effected by mixing various ratios of LP and DS in these media. The ratio of DS to LP in weight is expressed as DS/LP.

Turbidity, an indication of insoluble complex formation, was determined. One-half $\mathrm{ml}$ of the LP solution $(3 \mathrm{mg} / \mathrm{ml})$ in glycine medium of density 1.063 was placed in test tubes, and various amounts of dextran sulfate were added for DS/LP ranging from 0.0006 to 2.5 . The total volume of the mixtures

15) J. W. Gofman, F. Lindgren, H. Elliot, W. Mantz, J. Hewitt, B. Strisower and V. Herring, Science, 111, 166 (1950).

16) W.M. Sperry and M. Webb, J. Biol. Chem., 187, 97 (1950).

17) C. H. Fiske and Y. Subbarow, J. Biol. Chem., 66, 375 (1925). 
was then adjusted to $4 \mathrm{ml}$ by addition of the glycine medium. The contents were gently agitated and allowed to stand for 60 minutes. The optical densities of the suspensions were then determined with a Cary spectrophotometer at $650 \mathrm{~m} \mu$ against blank solutions containing the same amount of LP.

An apparent alteration in the ultracentrifugal behavior of LP caused by the formation of soluble DS-LP complex was studied by preparative ultracentrifugation in glycine and glucose media of density 1.063. Lipoprotein solutions in both the glycine and the glucose media $(24 \mathrm{mg}$ lipoprotein in final volume of $8 \mathrm{ml}$ ) were mixed with $24 \mathrm{mg}$ of DS. Before complete dissolution of DS, turbidity developed due to insoluble complex formation; however, the mixture cleared when all of the DS was dissolved. Eight $\mathrm{ml}$ of the LP solution in glucose or glycine media without DS was used as control. The DS-LP mixture and the controls were centrifuged in a Spinco Model 30.2 rotor at 30,000 r.p.m. at $5^{\circ} \mathrm{C}$ for 24 hours. For the ultracentrifugation in acidic media, DS-LP mixture and control in glycine or glucose media were adjusted to $\mathrm{pH} 4.0$ by addition of $1 \mathrm{~N}$ acetic acid. To locate boundary of the component in the preparative centrifuge tube, a hole was pierced in the bottom of each tube and 8 fractions were collected drop-by-drop, and these fractions were analyzed for LP by measuring the absorption due to carotenoid pigments at $400 \mathrm{~m} \mu$ and for DS by sulfur determination. 18

Ultracentrifugal analysis of the DS-LP mixture in glycine medium of density 1.063 was performed at 59,780 r.p.m. at $20^{\circ} \mathrm{C}$ in a Spinco Model E analytical ultracentrifuge with an acceleration time of 6 minutes. Mixtures of three different DS/LP ratios $(0.4,0.6$ and 1.0 ) with a DS concentration of $0.17 \%$ were examined.

\section{RESULTS AND DISCUSSION}

The addition of DS into a solution of LP in a glycine medium of density 1.063 to a DS/LP weight ratio of 1.0 and subsequent ultracentrifugation of the solution for 24 hours at $79,000 \times \mathrm{g}$ resulted in the sedimentation of LP together with DS (Fig. $1 \mathrm{~A}$ ). However, in the absence of DS, LP floated in this medium with a flotation rate $\left(S_{f}\right)$ of 6.0 due to its low hydrated density of 1.034 (Table I and Fig. 1 B). Thus, it is apparent that the association between LP and DS of hydrated density of 2.198 gave a soluble DS-LP complex with a hydrated density exceeding 1.063 .

Addition of sodium chloride, to the concentration of $0.1 \mathrm{M}$, into the mixture of $\mathrm{LP}$ and DS in the glycine medium caused dissociation of the complex, and LP floated to the top of the tube during centrifugation (Fig. $1 \mathrm{C})$. Dissociation of the complex was not observed at the concentration of sodium chloride below $0.05 \mathrm{M}$.

In the acidic glycine medium $(\mathrm{pH} 4)$ of density 1.063, DS-LP complex sedimented more rapidly than in neutral glycine medium as evident from observed sedimentation distance in the tube and formed a compact yellow layer at the bottom of the tube after 14 hours of centrifugation at $79,000 \times \mathrm{g}$. Furthermore, in this medium, the presence of $0.1 \mathrm{~m}$ sodium chloride did not alter the centrifugal

Table I. Some Physical Properties of Dextran Sulfate (DS) and $S_{f} 0-10$ LipopRoteIN (LP) IN PHOSPHATE BUFFER ( $\mathrm{pH} \mathrm{7.4,} \mu=0.1$ )

$\begin{array}{clccccc}\text { Materials } & S_{20, w}^{\circ} & S_{f} & {[\eta]} & \vec{V} & \text { Density } & \text { M } \times 10^{-5} \\ \text { DS } & 8.2^{1,} & - & 0.550 & 0.455 & 2.198 & 3.6^{2)} \\ \text { LP } & 7.3 & 6.0 & 0.029 & 0.967 & 1.034 & \left.23.2,3) 23.4^{4}\right\rangle\end{array}$

1) No correction for any possible charge effect was made.

2) Molecular weight of DS was calculated from that of original dextran $(180,000)$ and the sulfate content of DS.

3) From $S$ and $\bar{V}$ as sphere.

4) From $S$ and $[\eta](\mathrm{dl} / \mathrm{g})$.

18) F. Egami and N. Takahashi, Bull. Chem. Soc. Japan, 30, 442 (1957). 

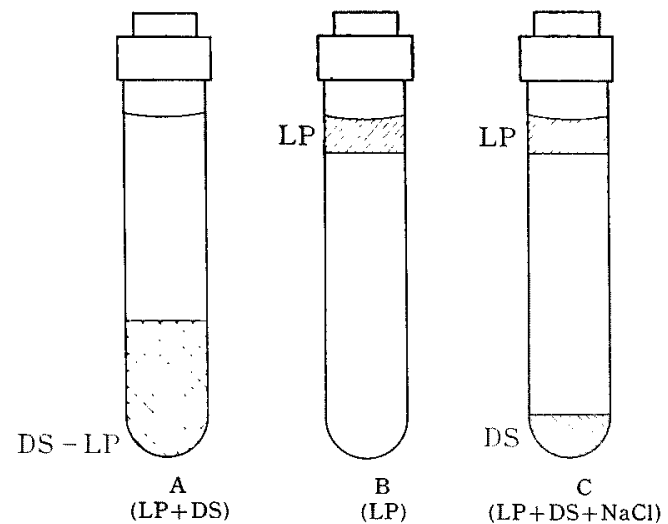

FIG. 1. Centrifugal Behavior of Soluble Dextran Sulfate (DS)-Low Density Lipoprotein (LP) Complex in Glycine Medium of Density 1.063

The abbreviations in the parentheses show the components in each tube prior to ultracentrifugation. Diagram A illustrates sedimentation of the LP as a soluble complex, DS-LP, and diagram $B$ shows flotation of the LP in the absence of DS. Separation of the two components (DS and LP) in the presence of $0.1 \mathrm{M}$ sodium chloride is shown by diagram $\mathrm{C}$.

behavior of the complex, indicating no dissociation of the complex. High stability of the complex at low $\mathrm{pH}$, in contrast to the dissociation of the complex in neutral glycine medium by the addition of salt, suggests that the linkage between DS and LP is ionic in nature.

It was suspected that the complex formation in glycine medium might be due to the specific ionic effect of dipolar glycine. However, the soluble complex is also formed in glucose media of density 1.063 , indicating that the complex formation is not affected by the properties of the medium used to elevate solvent density, provided the salt concentration in the medium is low.

The effect of DS/LP ratio on the formation of insoluble DS-LP complex in glycine medium of density 1.063 was similar to that in $0.1 \mathrm{~m}$ phosphate buffer of $\mathrm{pH} 7.4$ (Fig. 2). In both media, the maximum formation of insoluble complex was observed at the DS/LP ratio of 0.025 . When the $\mathrm{DS} / \mathrm{LP}$ ratio was 0.4 , no

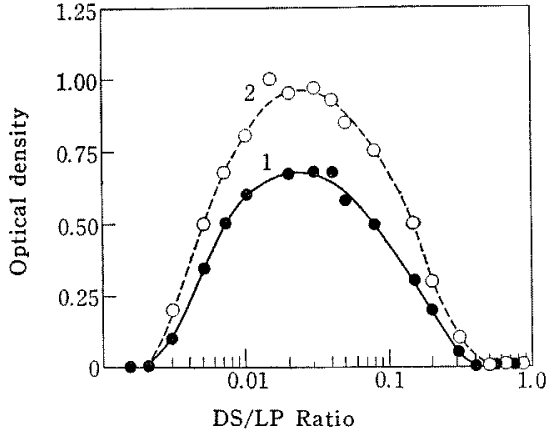

FIG. 2. Insoluble Complex Formation as a function of DS/LP Ratio.

Curves 1 and 2 show the development of turbidity, measured at $600 \mathrm{~m} \mu$, with changes of DS/LP ratio in glycine medium of density 1.063 and $0.1 \mathrm{M}$ phosphate buffer of $\mathrm{pH} 7.4$, respectively. The concentration of LP was $1.5 \mathrm{mg}$ in a total volume of $4 \mathrm{ml}$.

insoluble complex was formed. It is conceivable that, while the complexes may aggregate by extensive cross-linking of DS with LP molecules at low relative concentrations of DS, at high concentrations of DS, saturation of the binding sites of LP with DS molecules may result in formation of soluble units.

Ultracentrifugal analysis of the DS-LP mixture with a DS/LP ratio of 0.4 in glycine medium of density 1.063 revealed the presence of a single sedimenting boundary of soluble complex (Fig. $3 \mathrm{~A}$ ). The apparent sedimentation rate of this boundary was $2.7 \mathrm{~S}$ (Table II). The infinite Schlieren $(\mathrm{dn} / \mathrm{dc})$ pattern at the bottom of the cell is evidence of a pileup of the sedimented complex within a steep density gradient formed by glycine and, possibly, a trace of DS. Nevertheless, the single boundary of the complex indicated stoichiometric association of the two components at the DS/LS ratio of 0.4 . The approximate hydrated density and partial specific volume of the soluble complex were computed to be 1.22 and 0.82 , respectively, from the densities of DS and LP (Table I), assuming that these values were not affected by the high 


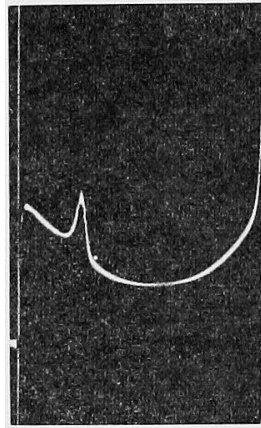

A
$(0.4)$

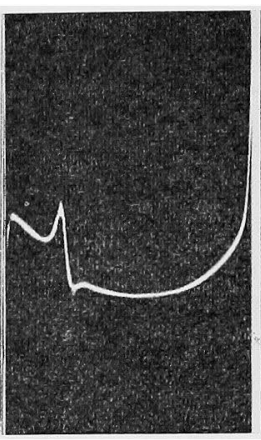

B

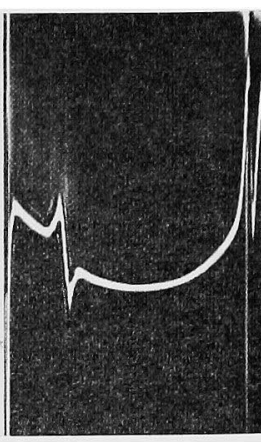

C

FIG. 3. Ultracentrigugal Analysis of Soluble DS-LP Complex in Glycine Medium of Density 1.063.

Patterns, A, B and C show the ultracentrifugal behaviors of DS-LP mixtures of the DS/LP ratios indicated in the parentheses. The concentration of DS in each mixtrure was $0.17 \%$. The pictures were taken at a bar angle of $55^{\circ}$, 85.5 minutes after a speed of 59,780 r.p.m. with an acceleration time of 6 minutes was reached.

concentration of glycine. The smallest possible unit of the soluble complex based on the DS/LP ratio of 0.4 consists of two or three DS molecules and one LP molecule and has a molecular weight of approximately $3 \times 10^{6}$, sum of molecular weights of the constituents (Table I). A rigid spherical molecule of this molecular weight and partial specific volume in the glycine medium is, however, expected to have a sedimentation rate of approximately $25 \mathrm{~S}$, which is far greater than the observed valus of $2.7 \mathrm{~S}$. The cause of this discrepancy may be shared by the charge effect of the complex and by a great structural deviation from spherical shape. Rather than being fixed rigidly on the spherical surface of the LP molecules, it is likely that DS molecules are expanded in the medium with only a partial contact with the LP molecules.

At DS/LP ratios above 0.4 , the presence of the soluble complex and free DS in glycine medium of density 1.063 resulted in complicated Schlieren patterns. At a DS/LP ratio of 0.6 , a sedimentation pattern with an asymmetric boundary associated with an inverted peak below the base line (Fig. $3 \mathrm{~B}$ ) was
Table II. Sedimentation Properties of the MIXTURE OF $S_{f}$ 0-10 LIPOPROTEIN (LP) AND DeXtran Sulfate (DS) in Glycine MEDIUM OF DENSITY 1.0631)

$\begin{array}{cccc}\begin{array}{c}\text { DS/LP ratio } \\ \text { of the } \\ \text { mixture }\end{array} & \mathrm{pH} & \begin{array}{c}\text { Schlieren } \\ \text { diagram of } \\ \text { boundary }\end{array} & \begin{array}{c}\text { Observed } \\ \text { sedimenta- } \\ \text { tion rate at } \\ 1.063\end{array} \\ 0.4 & 6.3 & \text { Symmetric } & 2.7 \mathrm{~S} \\ 0.6 & 6.3 & \text { Biphasic } & 2.5 \mathrm{~S} \\ 1.0 & 6.3 & \text { Biphasic } & 2.3 \mathrm{~S} \\ 1.0 & 4.0 & \text { Symmetric } & 3.0 \mathrm{~S}\end{array}$

1) The concentration of DS was $0.17 \%$ in each mixture.

produced. The apparent sedimentation rate of this biphasic boundary was less than that of the symmetrical boundary observed at the DS/LP ratio of 0.4 (Table II). Upon further increase in the ratio to 1.0 , the formation of the inverted peak was markedly increased (Fig. $3 \mathrm{C}$ ), and the apparent sedimentation rate of the biphasic boundary was further decreased. Furthermore, at the bottom of the cell, the sedimented complex formed a flotation-like pattern.

A biphasic sedimentation pattern was also 
observed for the undiluted serum and interpreted in terms of an unusual Johnston-Ogston effect by Gofman et al. ${ }^{191}$ The patterns of the present system might be the result of another type of Johnston-Ogston interaction which operates at both sedimentation and flotation boundaries of the system. Because of its high molecular weight, the sedimentation rate of the complex is greater than that of the free DS in the medium behind the trailing end of the sedimentation boundary of the latter, but smaller at the region ahead of the DS boundary where sedimentation of the low density complex is counteracted by a large buoyant force produced by free DS. Consequently, the complex is piled-up within the sedimenting DS boundary to give the biphasic Schlieren patterns (Fig. 4). On the other hand, at the bottom part of the cell the Johnston-Ogston effect is augmented by the composite density

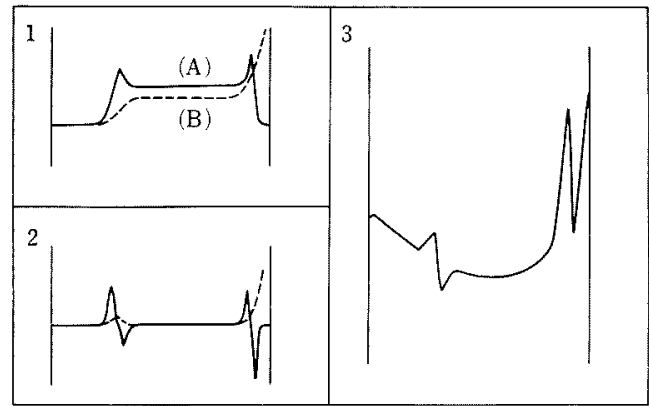

FIG. 4. Schematic Interpretation of the Biphasic Pattern Observed in the Analytical Ultracentrifugation of the Mixture of DS/LP Ratio of 1.0 in Glycine Medium of Density 1.063.

Diagrams 1 and 2 illustrate concentration distributions and concentration gradients of the complex (A) and free DS (B), respectively, as a function of the position in the cell at 117.5 minutes after attaining a speed of 59,780 r.p.m.

The curve of diagram 3 is the sum of the concentration gradient curves which would appear in the Schlieren optical system, taking into consideration the formation of a density gradient of the glycine medium.

19) J. W. Gofman, F. T. Lindgren, and H. Elliot, J. Biol. Chem., 179, 973 (1949). gradient formations of free DS and glycine medium. Within this steep gradient the migration of the complex changes from sedimentation to flotation, causing marked pile-up at a shifting position (Fig. 4); and, therefore, the Schlieren pattern resembled those obtained in density gradient equilibrium centrifugations.

The $\mathrm{pH}$ of the solution, in addition to the DS/LP ratio, seemed to influence the size of soluble complex. Addition of acetic acid to the $D S / L P$ mixture with a $D S / L P$ ratio of 1.0 reducing the $\mathrm{pH}$ to 4.0 caused disappearance of the inverted peak, resulting in the formation of a single sharp sedimenting boundary with an increased sedimentation rate (Table II). Since LP is isoelectric at $\mathrm{pH}$ 5.7, the lowering of the $\mathrm{pH}$ to 4.0 decreases the negative charges on the LP molecules. It is likely that at a lower $\mathrm{pH}$, more DS (Polyanion) approaches the binding sites of the LP molecules because of a lower ionic exclusion effect and, thus, reduced the amount of free DS.

Increase in the sedimentation rate of the DS-LP complex by lowering the relative concentration of free DS seemed to indicate that there was a concentration of free DS at which the sedimentation rates of the DS ard the complex became identical so that the JohnstonOgston effect was visually undetectable. Therefore, the single sedimentation boundary observed at a DS/LP ratio of 0.4 could be a composite Schlieren pattern of the complex and a trace of free DS rather than the complex alone. However, this would not significantly change the calculated properties of the soluble complex based on the DS/LP ratio of 0.4 .

In the present study the use of a glycine medium of density 1.063 which ensured the quantitative flotation of free lipoproteins and sedimentation of free DS allowed the study of the peculiar centrifugal behavior of DS-LP mixtures at high solvent density. Unlike salt solutions of the same density, the glycine 
medium did not cause the dissociation of course of this study. Appreciation is also DS-LP complex. Sedimentation of the LP given to Dr. Fred A. Kummerow for his along with DS in this medium provided direct encouragement while the research was in evidence of complex formation. progress.

This study was supported by PHS Research Acknowledgment The authors wish to thank Grant H-3063 from National Institute of Mrs. Chiga Janado and Mrs. Emelina M. Health, U.S. Public Health Service.

Villarreal for technical assistance during the 